\title{
A NOTE ON INFINITE LOOP SPACE MULTIPLICATIONS
}

\author{
RAINER M. VOGT
}

\begin{abstract}
A monoid $M$ is known to be abelian iff its multiplication $M \times$ $M \rightarrow M$ is a homomorphism. We prove the corresponding result for homotopyeverything $H$-spaces, e.g. infinite loop spaces: For a homotopy-everything $H$ space $X$ each $n$-ary operation $X^{n} \rightarrow X$ is a homotopy homomorphism, i.e. a homomorphism up to homotopy and all higher coherence conditions.
\end{abstract}

In [1] and [2] J. M. Boardman and I proved that an $H$-space $X$ is an infinite loop space iff its multiplication enjoys nice properties concerning associativity and commutativity. These properties were described in terms of universal algebra, and the necessary and sufficient condition for $X$ to be an infinite loop space essentially boils down to the fact that the morphism spaces $\mathcal{E}(n, 1)$ of the PROP $\mathcal{E}$ encoding the $H$-structure of $X$ be contractible (for the definition of a PROP see [2, Definition 2.44]). Dropping all unnecessary structure of a PROP, P. May in [4] introduced the simpler notion of an operad and obtained the corresponding result on infinite loop space structures more directly. Using his terminology we call $\varepsilon$ and $E_{\infty}$-PROP if each $\mathcal{E}(n, 1)$ is contractible and $\Sigma$-free if the operation of the symmetric group $\Sigma_{n}$ on $\mathcal{E}(n, 1)$ makes $\mathcal{E}(n, 1)$ a numerable principal $\Sigma_{n}$-space. An $E_{\infty}$-space $X$ is an $H$-space whose structure is given by an action of an $E_{\infty}$-PROP on $X$.

Let $\mathcal{E}$ be an $E_{\infty}$-PROP and $X$ an $\mathcal{E}$-space. There is a canonical product action of $\mathcal{E}$ on the $k$-fold product $X^{k}$. Each element $\chi \in \mathcal{E}(k, 1)$ defines a map $\chi: X^{k} \rightarrow X$. It is the purpose of this note to show

THEOREM. Suppose $\mathcal{E}$ is a $\Sigma$-free $E_{\infty}-P R O P$ or each $\mathcal{E}(n, 1)$ is $\Sigma_{n}$-equivariantly contractible. Let $\chi \in \mathcal{E}(k, 1)$ and $X$ be an $\mathcal{E}$-space. Then $\chi: X^{k} \rightarrow X$ can be extended to a homotopy $\mathcal{E}$-map in the sense of [2, Definition 4.2].

T. Lada tried in [3] to prove a result of this kind but only succeeded in the case $k=2$ and $\mathcal{E}=\mathcal{Q}$, the little cubes PROP of $[2,2.49]$. His proof is given by a number of explicit formulas depending on the geometry of the spaces of little cubes. Our proof of the theorem is an easy consequence of the theory of [1] and [2]. We use the terminology of [2, Chapter III, IV].

Let $\mathcal{E}$ be an arbitrary PROP and $X$ and $\mathcal{E}$-space. Then $\mathcal{E}$ operates on $X^{k}$ by

$$
\alpha:\left(X^{k}\right)^{n} \stackrel{\tau_{n, k}}{\rightarrow}\left(X^{n}\right)^{k} \stackrel{\alpha^{k}}{\rightarrow} X^{k},
$$

$\alpha \in \mathcal{E}(n, 1)$, where $\tau_{n, k}$ is the homeomorphism

$$
\left(\left(x_{11}, \ldots, x_{1 k}\right), \ldots,\left(x_{n 1}, \ldots, x_{n k}\right)\right) \rightarrow\left(\left(x_{11}, \ldots, x_{n 1}\right), \ldots,\left(x_{1 k}, \ldots, x_{n k}\right)\right) .
$$

Received by the editors May 17, 1981.

1980 Mathematics Subject Classification. Primary 55P47.

Key words and phrases. Homotopy-everything $H$-spaces, infinite loop space, homotopy homomorphism. 
To prove the theorem we need an action of $H W\left(\mathcal{E} \otimes \mathcal{L}_{1}\right)$ extending $\chi$ and the actions on $X^{k}$ and $X$. The idea is to apply the Lifting Theorem [2, Theorem 3.7] with $\mathcal{V} \subset H W\left(\mathcal{E} \otimes \mathcal{L}_{1}\right)$ being the subcategory generated under composition and $\oplus$ by $W(\mathcal{E} \otimes\{0\}), W(\mathcal{Q} \otimes\{1\})$ and the single morphism $\left(\mathrm{id}_{1} \otimes(0 \rightarrow 1)\right)$. To minimize notation we denote the objects $(n, 0)$ and $(n, 1)$ in $\mathcal{E} \times \mathcal{L}_{1}$ by $n$ respectively $n^{\prime}$. For $C$ we take the PROP $S \otimes \mathcal{L}_{1}$, where $S$ is the PROP of abelian monoids, i.e. $S(n, 1)$ consists of a single point for all $n$. We have to construct an appropriate PROP $D$ acting on the pair $\left(X^{k}, X\right)$ extending the $\mathcal{E}$-action on $X^{k}$ and on $X$ and the map $\chi$, yielding a commutative diagram:

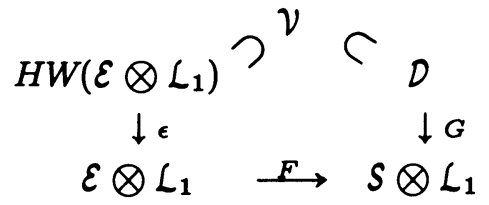

Here $F$ and $G$ are the uniquely determined PROP-functors. If each $D(n, 1), D\left(n, 1^{\prime}\right)$ and $D\left(n^{\prime}, 1^{\prime}\right)$ is contractible (respectively $\Sigma_{n}$-equivariantly contractible), and $\varepsilon$ is $\Sigma$-free (respectively arbitrary) the theorem is proved.

Construction of $D$. It suffices to specify

$$
D(n, 1):=\mathcal{E}(n, 1), \quad D\left(n, 1^{\prime}\right):=\mathcal{E}(n k, 1), \quad D\left(n^{\prime}, 1^{\prime}\right):=\mathcal{E}(n, 1),
$$

the composite of a morphism $\alpha$ in these spaces with a permutation $\pi \in \Sigma_{n}$, and of $\alpha$ with an $n$-fold sum $\beta_{1} \oplus \ldots \oplus \beta_{n}$ with $\beta_{i}$ in the appropriate spaces (A). As long as we stick in the full subcategories of objects $n$ respectively $n^{\prime}$ these compositions are given by the composition in $\mathcal{E}$. If $\alpha \in D\left(n^{\prime}, 1^{\prime}\right)$ and $\beta_{i} \in D\left(n_{i}, 1^{\prime}\right)$ the composition is again the one in $\mathcal{E}$. It remains to define $\alpha \circ \pi$ and $\alpha \circ\left(\beta_{1} \otimes \cdots \otimes \beta_{n}\right)$ for $\alpha \in D\left(n, 1^{\prime}\right)=\mathcal{E}(n k, 1), \beta_{i} \in D\left(n_{i}, 1\right)=\mathcal{E}\left(n_{i}, 1\right)$ :

$$
\begin{gathered}
\alpha \circ \pi=\alpha \circ(\pi \oplus \cdots \oplus \pi), \\
\alpha \circ\left(\beta_{1} \oplus \cdots \oplus \beta_{n}\right)=\alpha \circ\left[\left(\beta_{1} \oplus \cdots \oplus \beta_{n}\right) \oplus \cdots \oplus\left(\beta_{1} \oplus \cdots \oplus \beta_{n}\right)\right],
\end{gathered}
$$

where on the right side we have composition in $\mathcal{E}$ with $k$ summands $\pi$ respectively $\left(\beta_{1} \oplus \cdots \oplus \beta_{n}\right)$.

The operation of $D$ on the pair $\left(X^{k}, X\right)$ is given as follows.

$\alpha \in D(n, 1)=\mathcal{E}(n, 1)$ operates as $\alpha \circ \tau_{n, k}:\left(X^{k}\right)^{n} \rightarrow X^{k}$,

$\alpha \in D\left(n, 1^{\prime}\right)=\mathcal{E}(n k, 1)$ operates as $\alpha \circ \tau_{n, k}:\left(X^{k}\right)^{n} \rightarrow X$,

$\alpha \in D\left(n^{\prime}, 1^{\prime}\right)=\mathcal{E}(n, 1)$ operates as $\alpha: X^{n} \rightarrow X$.

Obviously, $D$ satisfies all the requirements listed above, which proves the theorem.

\section{REFERENCES}

1. J. M. Boardman and R. M. Vogt, Homotopy-everything $H$-spaces, Bull. Amer. Math. Soc. 74 (1968), 1117-1122.

2. - Homotopy invariant algebraic structures on topological spaces, Lecture Notes in Math., vol. 347, Springer-Verlag, Berlin and New York, 1973.

3. T. Lada, An operad action on infinite loop space multiplication, Canad. J. Math. 29 (1977), 1208-1216.

4. J. P. May, The geometry of iterated loop spaces, Lecture Notes in Math., vol. 271, SpringerVerlag, Berlin and New York, 1972.

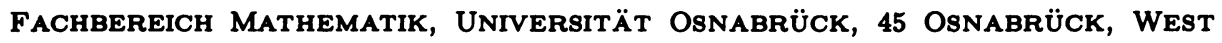
GERMANY 